\title{
Microhairs of Indian Eragrostis (Poaceae) and their taxonomic significance
}

\author{
C. P. Vivek ${ }^{1}$, G. Gnanasekaran², G. V. S. Murthy ${ }^{2 *}$ and V. J. Nair ${ }^{2 \otimes}$ \\ 'Botanical Survey of India, Andaman and Nicobar Regional Centre, Port Blair - 744102, Andaman \& Nicobar Islands \\ 2Botanical Survey of India, Southern Regional Centre, TNAU Campus, Coimbatore - 641003, Tamil Nadu, India \\ 凶Corresponding author: gvs.23.murthy@gmail.com
}

\section{भारतीय इराग्रोसटिस (पोएसी) के सूक्ष्म बाल एवं उनका वर्गीकरण महत्व}

\author{
सी. पी. विवेक, जी. ज्ञानसेकरण, जी. वी. एस. मूर्ति एवं वी. जे. नायर
}

\section{सारांश}

स्कैनिंग इलेक्ट्रॉन सूक्ष्मदर्शी द्वारा भारत में 44 इराग्रोसटिस प्रजातियों के पत्ती की सतह पर सूक्ष्म बाल की आकारिकी पर किया गया अध्ययन प्रस्तुत है। सूक्ष्म बाल के तीन अलग प्रकार अर्थात कलोरिडोइड, पानिकोइड और एनिआपोगोन (मध्यवर्ती) देखे गए हैं एवं उनकी विशेषताएं सारणीबद्ध हैं।

\begin{abstract}
The morphology of microhairs on leaf surfaces of 44 Eragrostis species from India is studied using Scanning Electron Microscope (SEM) and the results are presented. Three distinct types of microhairs have been observed viz. chloridoid, panicoid and enneapogon (intermediate) and their features are tabulated.
\end{abstract}

\section{Keywords: Eragrostis, India, Leaf Surface, Microhairs INTRODUCTION}

Eragrostis is a cosmopolitan genus, largest in the subfamily Chloridoideae with about 423 species distributed in the tropical and subtropical regions of the world (Clayton \& Renvoize, 1986; Veldkamp, 2002; Ingram, 2010; GiraldoCanas \& al., 2012). According to Bor (1960), there are 39 species of this genus occurring in India and Karthikeyan \& al. (1989) considered this number to be 36 . With the addition of recently published new species from India (Vivek \& al., 2012, 2013a, 2013b \& 2013c), now the total infrageneric taxa of this genus in India is 44 species and 9 varieties. Out of this total number, 5 species and 4 varieties are endemic to the country, viz. E. ciliaris var. brachystachya Boiss., E. ciliaris var. clarkei Stapf, E. collinensis Vivek, Murthy \& V. J. Nair, E. deccanensis Bor, E. jainii Vivek, Murthy \& V.J. Nair, E. henryi Vivek, Murthy \& V. J. Nair, E. minor var. rajasthanensis Vivek,
Murthy \& V. J. Nair, E. santapaui K. G. Bhat \& Nagendran, and E. tremula var. gajanandii Singh, Bala \& Purohit.

Microhair is one of the significant characters of high taxonomic value in the family Poaceae. It is a specialized 2-celled trichome, commonly found on the surface of leaves but also on other parts of plants (Tateoka \& al., 1959; Metcalfe, 1960). Microhair is not present in the subfamily Pooideae, however is present in all other subfamilies of Poaceae (Johnston \& Watson, 1976; Watson \& al., 1985). It is distributed usually on culms, upper leaf surface, lemmas, paleas and lodicules in the genus Eragrostis, however, few in numbers (Tateoka \& al., 1959; Metcalfe, 1960), and relatively more on the lower part of the leaves. Depending on the proportional length and shape of their basal and apical cells and thickness of the cell wall, it is categorized into three types: chloridoid, panicoid and enneapogon (intermediate). Chloridoid type has short, broad and thick-walled cap cell whereas in panicoid type the 
cap cell is relatively longer, narrow and thin-walled (Plate1). The ennaepogon (intermediate) type possesses remarkably long (up to $400 \mu \mathrm{m}$ ) basal cell and elongated ovoid cap cell. The subfamily Chloridoideae is characterized by having chloridoid type of microhair (Amarasinghe \& Watson, 1990). The genus Eragrostis belonging to this subfamily is an exception where chloridoid, panicoid and ennaepogon (intermediate) types of microhairs are seen. Chloridoid however is the most common type (Tateoka \& al., 1959).

\section{MATERIALS AND METHODS}

The morphology of microhair on leaf surfaces of 44 species of Eragrostis from India was examined using a Scanning Electron Microscope (SEM). The mature leaves of each species were soaked in water and cut into quadrangular pieces of the size of about $0.5 \mathrm{~cm}^{2}$. Further, their surface was cleaned with ethyl alcohol. Later, the leaves were mounted directly on stubs using double-sided adhesive tape and they were sputter-coated for 60 seconds with gold-palladium mixture in a SC7620 Sputter Coater (EMITECH). After coating, leaves were examined with a Scanning Electron Microscope (Evo M18, Carl Zeiss), using an accelerating voltage of 5 to $15 \mathrm{KV}$. Multiple micrographs and measurements were made using the Image Tool software.

\section{RESULTS AND DISCUSSION}

Out of 44 species studied for the microhair morphology of Indian Erograstis, 26 species were found to have chloridoid and 5 panicoid types of microhairs. In 3 species (E. amabilis, E. ciliaris, E. jainii) of both chloridoid and panicoid types and in another 3 species (E. curvula, E. pilosa, E. tef), both ennaepogon (intermediate) and chloridoid types were found within the same species. A total of 22 species were studied for their microhair morphology for the first time with SEM, which includes 6 endemic species to India: E. collinensis, E. deccanensis, E. henryi, E. jainii, E. nilgiriensis and E. santapaui. Table 1 shows the name of species and type of microhairs based on the present study and earlier published works (Amarasighe \& Watson, 1990; Giraldo-Canas \& al., 2012).

During the present study, majority of the species were examined using freshly collected specimens since microhairs are usually caducous in the preserved herbarium specimens. However, for a total of six species namely E. ferruginea, E. lehmanniana, E. multicaulis, E. plana, E. subsecunda and E. trichodes, the present authors could not locate any microhairs under the microscope as they were based on old herbarium specimens housed at CAL and MH. In addition, it is also noted here that these species have not been studied by the earlier workers. Therefore, they are represented as 'not seen' in the table.

Table 1. Different types of microhairs encounted in genus Eragrostis

\begin{tabular}{|c|c|c|}
\hline NAME OF SPECIES & TYPE OF MICROHAIRS & SPECIMENS STUDIED \\
\hline E. amabilis (L.) Wight \& Arn. & $\begin{array}{l}\text { Chloridoid \& Panicoid (Amarasinghe \& Watson, } \\
\text { 1990; Giraldo-Canas \& al., } \\
\text { 2012); Chloridoid (present study) }\end{array}$ & $\begin{array}{l}\text { N.P. Balakrishnan 444; K. Subramanyam 6905; C. P. } \\
\text { Vivek } 126114(\mathrm{MH})\end{array}$ \\
\hline E. aspera (Jacq.) Nees & Chloridoid (present study) & V. Narayanaswamy 4516; C.P. Vivek 126148 (MH) \\
\hline E. atrovirens (Desf.) Trin. ex Steud. & $\begin{array}{l}\text { Panicoid (Amarasinghe \& Watson, } \\
\text { 1990; Giraldo-Canas \& al., 2012; present study) }\end{array}$ & $\begin{array}{l}\text { K. Subramanyam } 2908 \text { (CAL); J.L. Ellis 23838; C. P. } \\
\text { Vivek } 126154(\mathrm{MH})\end{array}$ \\
\hline E. burmanica Bor & Chloridoid (present study) & N.L. Bor 15438 (CAL) \\
\hline $\begin{array}{l}\text { E. cilianensis (All.) Vignolo Lut. ex } \\
\text { Janch. }\end{array}$ & $\begin{array}{l}\text { Chloridoid (Amarasinghe \& Watson, } \\
\text { 1990; Giraldo-Canas \& al., 2012) }\end{array}$ & E. Vajravelu 27574 (CAL \& MH) \\
\hline E. ciliaris (L.) R. Br. & $\begin{array}{l}\text { Panicoid (Amarasinghe \& Watson, } \\
\text { 1990; Giraldo-Canas \& al., 2012); } \\
\text { Chloridoid (present study) }\end{array}$ & $\begin{array}{l}\text { B.V. Shetty 3308; A.R.K. Sastry } 7621 \text { (CAL); K.M. } \\
\text { Sebastine } 9819 \text { (CAL \& MH) }\end{array}$ \\
\hline E. ciliata (Roxb.) Nees & Chloridoid (present study) & $\begin{array}{l}\text { V. Narayanswami 4597; K.C. Jacob 17097; D. Daniel } \\
\text { \& S. R. Raju } 20431(\mathrm{MH})\end{array}$ \\
\hline E. coarctata Stapf & Chloridoid (present study) & $\begin{array}{l}\text { V. Narayanaswami 5963; R. Chandrasekaran 98910; } \\
\text { M. Mohanan } 101241(\mathrm{MH})\end{array}$ \\
\hline $\begin{array}{l}\text { E. collinensis Vivek, G. V. S. Murthy \& } \\
\text { V. J. Nair }\end{array}$ & Chloridoid (present study) & $\begin{array}{l}\text { V. Narayanaswamy } 4373 ; \text { K. Vivekananthan } 46598 \text {; } \\
\text { K. Vivekananthan } 42951(\mathrm{MH})\end{array}$ \\
\hline E. cumingii Steud. & Panicoid (Amarasinghe \& Watson, 1990) & $\begin{array}{l}\text { V. S. Ramachandran } 63327 \text { (MH); M. Mohanan } \\
62649 \text { (CAL \& MH) }\end{array}$ \\
\hline E. curvula (Schrad.) Nees & $\begin{array}{l}\text { Intermediate (Amarasinghe \& Watson, } \\
\text { 1990; Giraldo-Canas \& al., 2012); } \\
\text { Chloridoid (present study) }\end{array}$ & $\begin{array}{l}\text { D. Daniel Sunder Raj s.n., MH acc. no. } 94330 \text { \& } \\
\text { 94331; Asst. Surgeon s.n., MH acc. no. } 97166\end{array}$ \\
\hline
\end{tabular}




\begin{tabular}{|c|c|c|}
\hline NAME OF SPECIES & TYPE OF MICROHAIRS & SPECIMENS STUDIED \\
\hline E. deccanensis Bor & Chloridoid (present study) & K.C. Jacob 471; V. Narayanaswami 3027 (MH) \\
\hline E. diarrhena (Schult.) Steud. & Chloridoid (present study) & $\begin{array}{l}\text { M. Chandrabose } 28838 ; 28841 \text {; C.P. Vivek } 126132 \text {; } \\
\text { C.P. Vivek } 126139(\mathrm{MH})\end{array}$ \\
\hline E. diplachnoides Steud. & Chloridoid (present study) & J.L. Ellis 15754 (MH); Hemadri 102341 (CAL) \\
\hline E. ferruginea (Thunb.) P. Beauv. & Not seen & V. Komorov $174 ; 2743 ; 4288(\mathrm{CAL})$ \\
\hline E. gangetica (Roxb.) Steud. & $\begin{array}{l}\text { Panicoid (Giraldo-Canas \& al., 2012; } \\
\text { present study) }\end{array}$ & $\begin{array}{l}\text { K. Subramanyam 4551; G.V. Subbarao 22511; R. } \\
\text { Ansari } 74308(\mathrm{MH})\end{array}$ \\
\hline $\begin{array}{l}\text { E. henryi Vivek, G. V. S. Murthy \& V. } \\
\text { J. Nair }\end{array}$ & Chloridoid (present study) & A. N. Henry 61563 (CAL \& MH) \\
\hline $\begin{array}{l}\text { E. jainii Vivek, G. V. S. Murthy \& V. } \\
\text { J. Nair }\end{array}$ & Panicoid \& Chloridoid (present study) & $\begin{array}{l}\text { V.S. Ramachandran 52200; R. Ansari 64888; V. S. } \\
\text { Ramachandran } 65258(\mathrm{MH})\end{array}$ \\
\hline E. japonica (Thunb.) Trin. & Chloridoid (present study) & $\begin{array}{l}\text { K.N. Subramanyan } 1511 \text { (FRC); C.P. Vivek \& C. } \\
\text { Kalidass } 126149(\mathrm{MH})\end{array}$ \\
\hline E. lehmanniana Nees & Not seen & L.H. Harvey \& J.C. Elliott 7293; 7318 (CAL) \\
\hline E. macilenta (A. Rich.) Steud. & Chloridoid (present study) & A. Pappi $2672(\mathrm{CAL})$ \\
\hline E. maderaspatana Bor & Chloridoid (present study) & $\begin{array}{l}\text { K.N. Subramanyan } 899 \text { (FRC); V. Balasubraman- } \\
\text { yam 1514; S.R. Raju } 6184(\mathrm{MH})\end{array}$ \\
\hline E. minor Host & $\begin{array}{l}\text { Chloridoid (Amarasinghe \& Watson, } \\
\text { 1990; present study) }\end{array}$ & $\begin{array}{l}\text { V. Balasubramanyam 2060; V. Narayanaswami } \\
3260(\mathrm{MH})\end{array}$ \\
\hline E. multicaulis Steud. & Not seen & $\begin{array}{l}\text { G. King s.n., CAL acc. no. 542146; G. King s.n., } \\
\text { CAL acc. no. } 542148\end{array}$ \\
\hline E. nigra Nees ex Steud. & $\begin{array}{l}\text { Chloridoid (Amarasinghe \& Watson, } \\
\text { 1990; present study) }\end{array}$ & $\begin{array}{l}\text { K.C. Jacob 20488; C.P. Vivek 126135; C.P. Vivek } \\
\text { 126136; C.P. Vivek } 126155 \text { (MH) }\end{array}$ \\
\hline E. nilgiriensis Vivek, G.V.S. Murthy \& & Chloridoid (Vivek, G. V. S. Murthy \& & K. M. Sebastine 4764; J. S. Gamble 20841; C. P. \\
\hline $\begin{array}{l}\text { E. nutans (Retz.) Nees ex Wight \& } \\
\text { Arn. }\end{array}$ & Chloridoid (present study) & $\begin{array}{l}\text { V. Balasubramanyam 1496; K. Subramanyam 3809; } \\
\text { C. N. Mohanan } 55729 \text { (MH) }\end{array}$ \\
\hline E. pappiana Chiov. & Chloridoid (present study) & Coll. ign., 66941 (BSI) \\
\hline E. papposa (Duf.) Steud. & Chloridoid (present study) & $\begin{array}{l}\text { B. Krishna 1785; S.K. Lucksome 2824; D.C.S. Raju } \\
7321 \text { (BSHC) }\end{array}$ \\
\hline E. pilosa (L.) P. Beauv. & $\begin{array}{l}\text { Intermediate (Amarasinghe \& Watson, } \\
\text { 1990; Giraldo-Canas \& al., 2012); } \\
\text { Chloridoid (present study) }\end{array}$ & $\begin{array}{l}\text { C.N. Mohanan 77994; C.P. Vivek \& S. Arumugam } \\
\text { 126131; C.P. Vivek } 126140(\mathrm{MH})\end{array}$ \\
\hline E. plana Nees & Not seen & K. C. Jacob $6353(\mathrm{MH})$ \\
\hline E. riparia (Willd.) Nees & Chloridoid (present study) & $\begin{array}{l}\text { G. V. Subba Rao 19584; A. N. Henry } 47096 \text { (CAL); } \\
\text { C.P. Vivek } 126122(\mathrm{MH})\end{array}$ \\
\hline E. rottleri Stapf & Not seen & - \\
\hline E. santapaui K.G. Bhat \& Nagendran & Chloridoid (present study) & $\begin{array}{l}\text { K. Gopalakrishna Bhat 794; S.R. Ramesh \& P. } \\
\text { Prakash } 5281 \text { (CAL) }\end{array}$ \\
\hline E. schweinfurthii Chiov. & Chloridoid (present study) & C.P. Vivek $126157(\mathrm{MH})$ \\
\hline E. subsecunda (Lam.) Four. & Not seen & P.V. Sreekumar $67646(\mathrm{MH})$ \\
\hline E. superba Peyr. & $\begin{array}{l}\text { Chloridoid (Amarasinghe \& Watson, } \\
\text { 1990; present study) }\end{array}$ & K. Subramanyam 3538 (MH) \\
\hline E. tef (Zucc.) Trotter & $\begin{array}{l}\text { Intermediate (Amarasinghe \& Watson, } \\
\text { 1990); Chloridoid (present study) }\end{array}$ & Coll. ign., $13983(\mathrm{MH})$ \\
\hline $\begin{array}{l}\text { E. tenuifolia (A. Rich.) Hochst. ex } \\
\text { Steud. }\end{array}$ & $\begin{array}{l}\text { Chloridoid (Amarasinghe \& Watson, 1990; } \\
\text { Giraldo-Canas \& al., 2012; present study) }\end{array}$ & $\begin{array}{l}\text { V. S. Ramachandran 62035; C.P. Vivek 126134; C. P. } \\
\text { Vivek } 126156(\mathrm{MH})\end{array}$ \\
\hline E. tremula Hochst. ex Steud. & Chloridoid (present study) & $\begin{array}{l}\text { V. Narayanaswami } 4516 \text {; G.V. Narayana 6313; V.S. } \\
\text { Ramachandran } 65259(\mathrm{MH})\end{array}$ \\
\hline E. trichodes (Nutt.) Wood & Not seen & Le Roy H. Harvey 8324; Harvey 8326 (CAL) \\
\hline E. unioloides (Retz.) Nees ex Steud. & $\begin{array}{l}\text { Panicoid (Amarasinghe \& Watson, } \\
\text { 1990; present study) }\end{array}$ & $\begin{array}{l}\text { V.J. Nair 59957; C. P. Vivek 126101, 126102; C. P. } \\
\text { Vivek } 126142(\mathrm{MH})\end{array}$ \\
\hline E. viscosa (Retz.) Trin. & Chloridoid (present study) & $\begin{array}{l}\text { C. P. Vivek, 126111; C. P. Vivek 126150; C. P. Vivek } \\
126158(\mathrm{MH})\end{array}$ \\
\hline E. zeylanica Nees \& Mey. & Panicoid (present study) & $\begin{array}{l}\text { N.P. Balakrishnan } 661 \text { (CAL); V. S. Ramachandran } \\
60035 \text { (CAL \& MH) }\end{array}$ \\
\hline
\end{tabular}



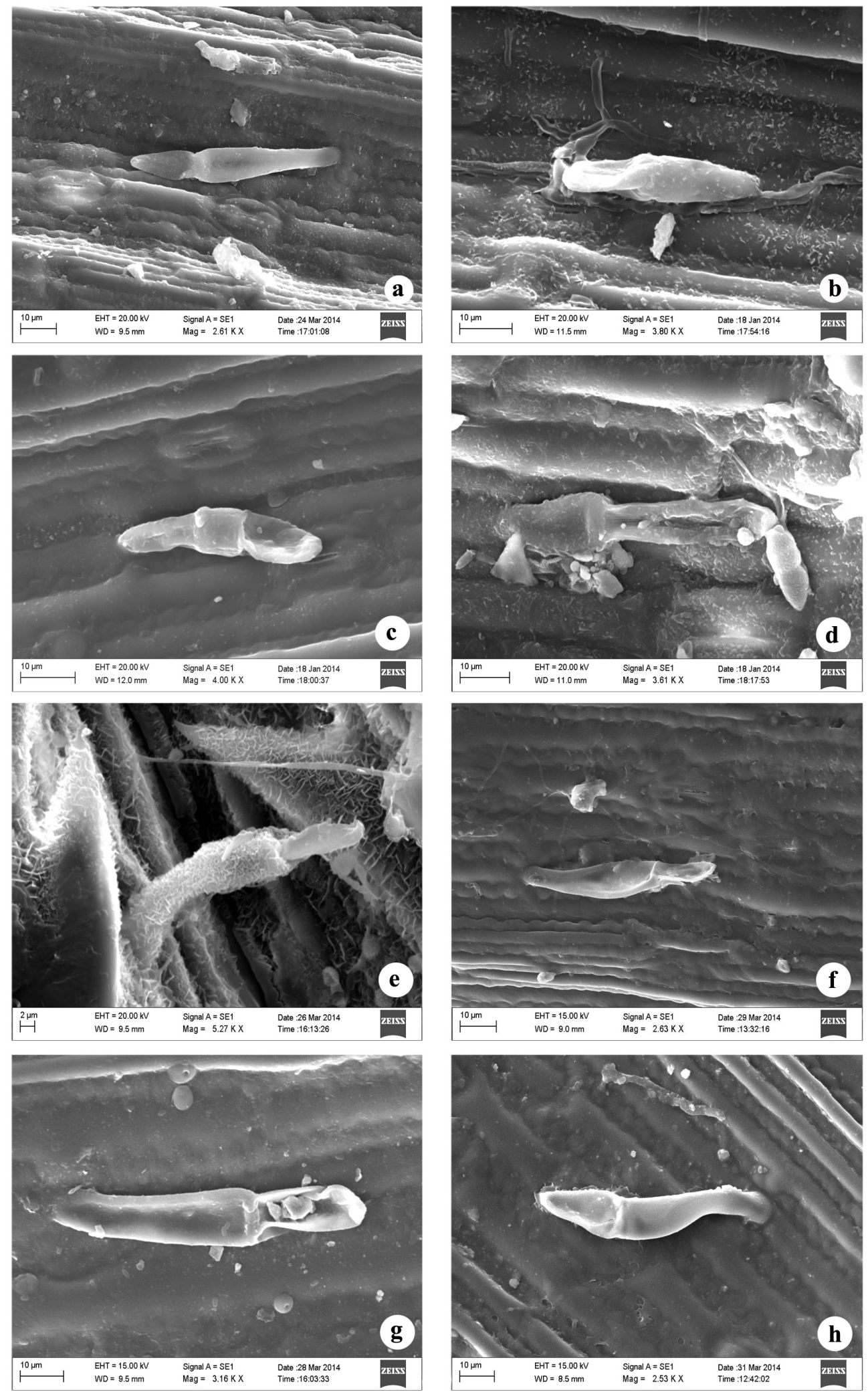

Plate 1. SEM feature of microhairs of: a. Eragrostis amabilis (L.) Wight \& Arn.; b \& c. E. aspera (Jacq.) Nees; d. E. atrovirens (Desf.) Trin. ex Steud.; e. E. burmanica Bor; f. E. ciliaris (L.) R.Br.; g. E. ciliata (Roxb.) Nees; h. E. coarctata Stapf. 

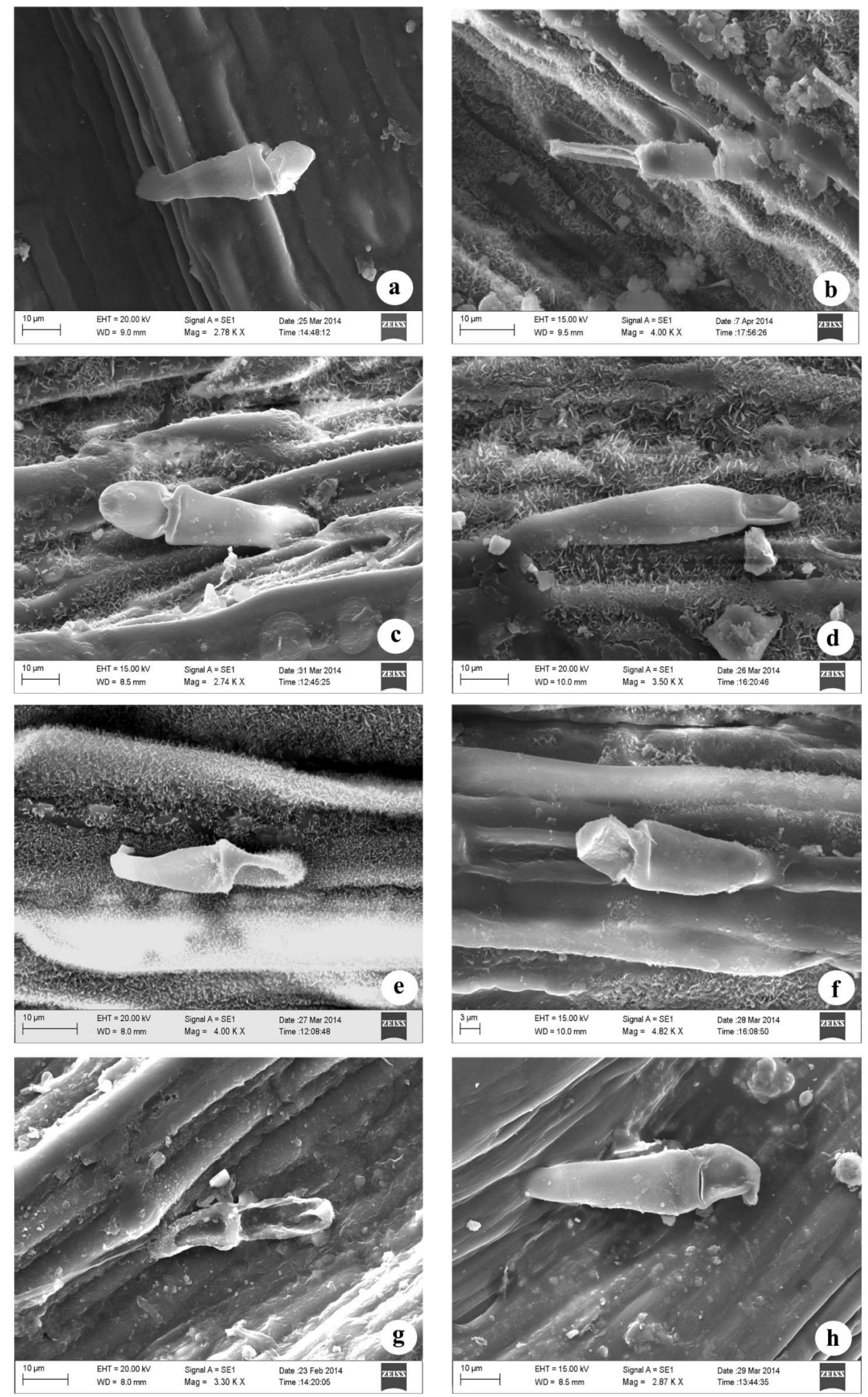

Plate 2. SEM feature of microhairs of: a. E. collinensis Vivek, G.V.S. Murthy \& V.J. Nair; b. E. cumingii Steud.; c. E. curvula (Schrad.) Nees; d. E. deccanensis Bor; e. E. diarrhena (Schult.) Steud.; f. E. diplachnoides Steud.; g. E. gangetica (Roxb.) Steud.; h. E. henryi Vivek, G.V.S. Murthy \& V.J. Nair. 

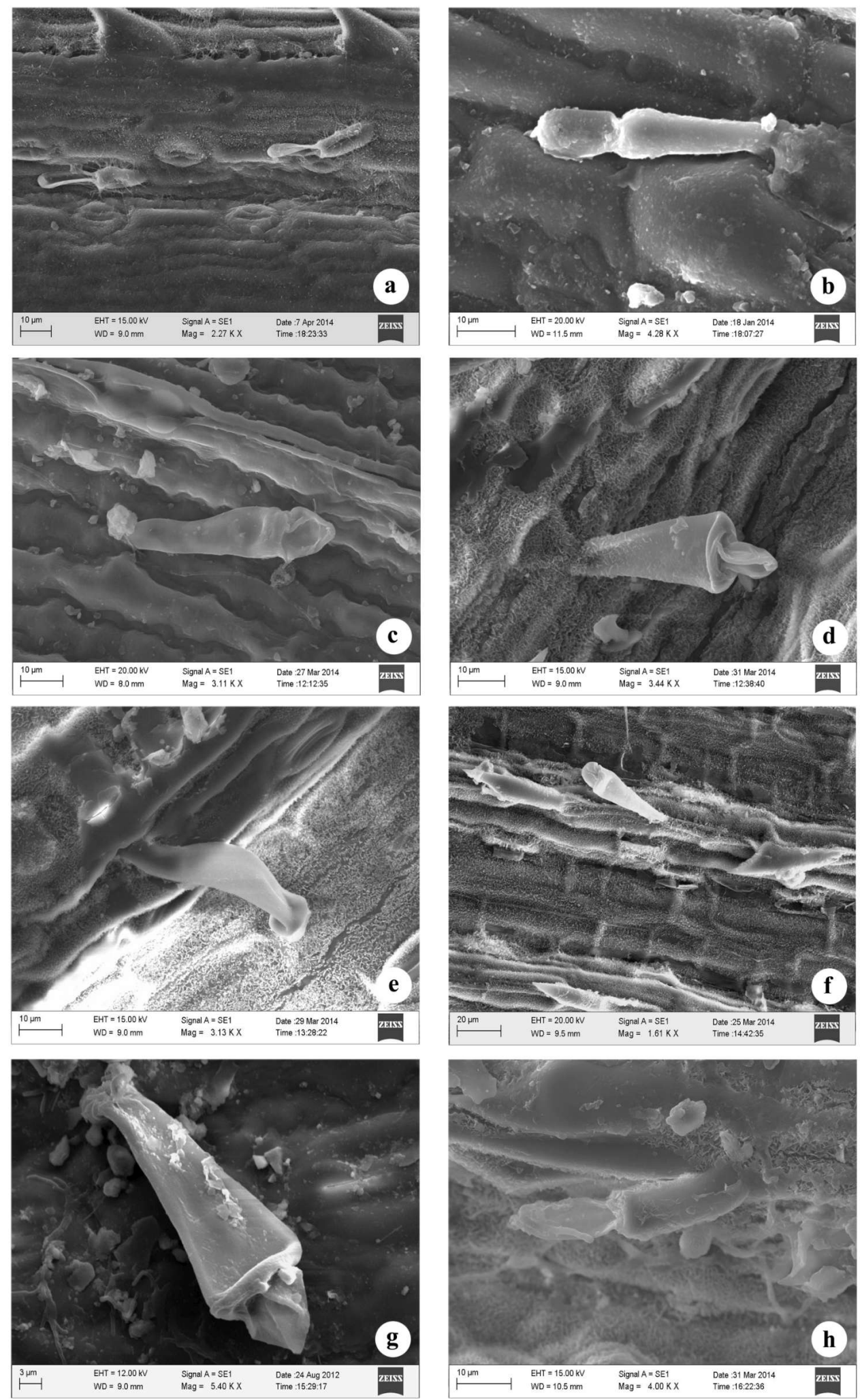

Plate 3. SEM feature of microhairs of: a. E. jainii Vivek, G.V.S. Murthy \& V.J. Nair; b. E. japonica (Thunb.) Trin.; c. E. macilenta (A. Rich.) Steud.; d. E. maderaspatana Bor; e. E. minor Host; f. E. nigra Nees ex Steud.; g. E. nilgiriensis Vivek G.V.S. Murthy \& V.J. Nair; h. E. nutans (Retz.) Nees ex Wight \& Arn. 

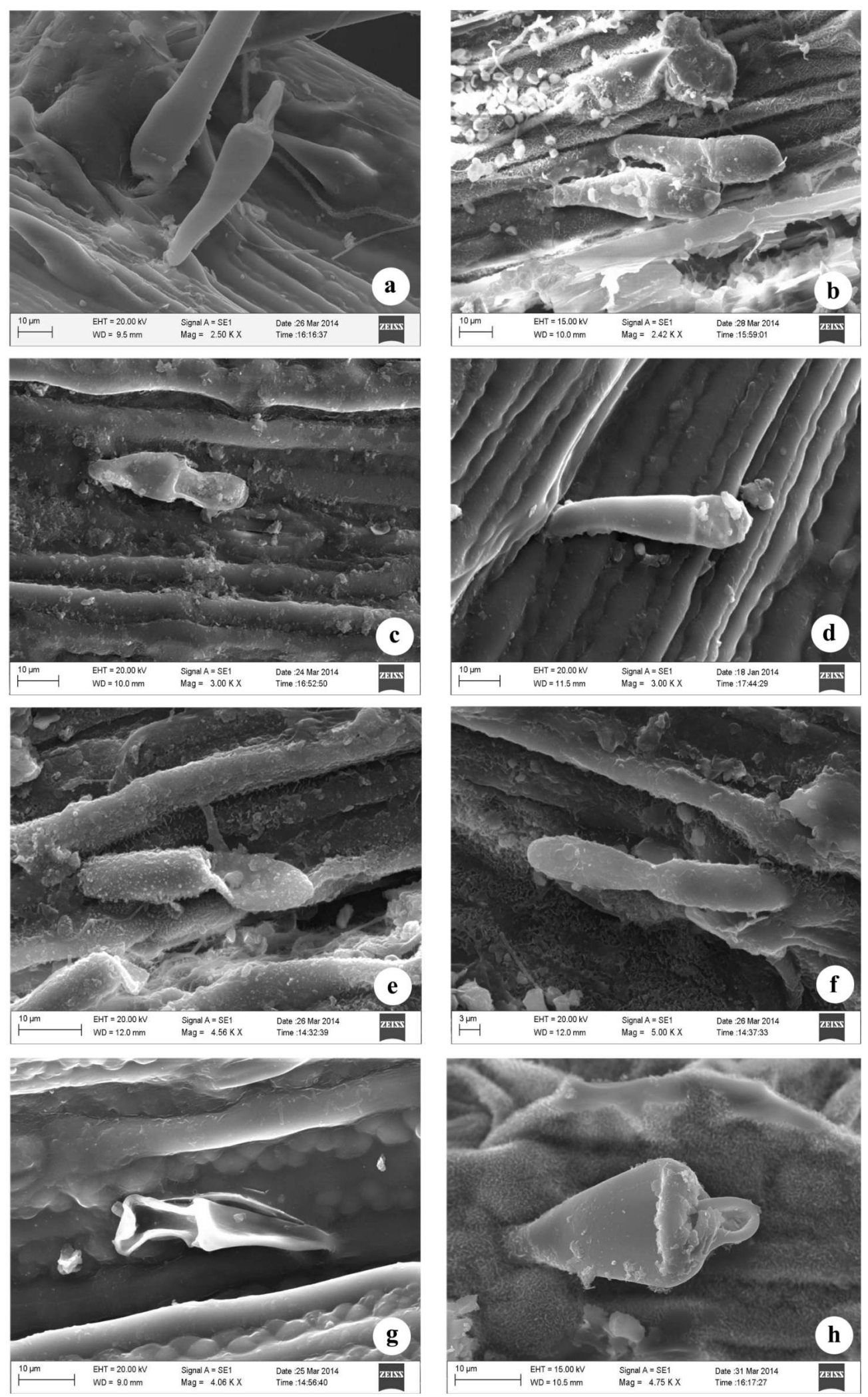

Plate 4. SEM feature of microhairs of: a. E. pappiana chiov.; b. E. papposa (Duf.) Steud.; c. E. pilosa (L.) P. Beauv.; d. E. riparia (Willd) Nees; e \& f. E. santapaui K.G Bhat \& Nagendran; g. E. schweinfrthii Chiov.; h. E. superba Peyr. 

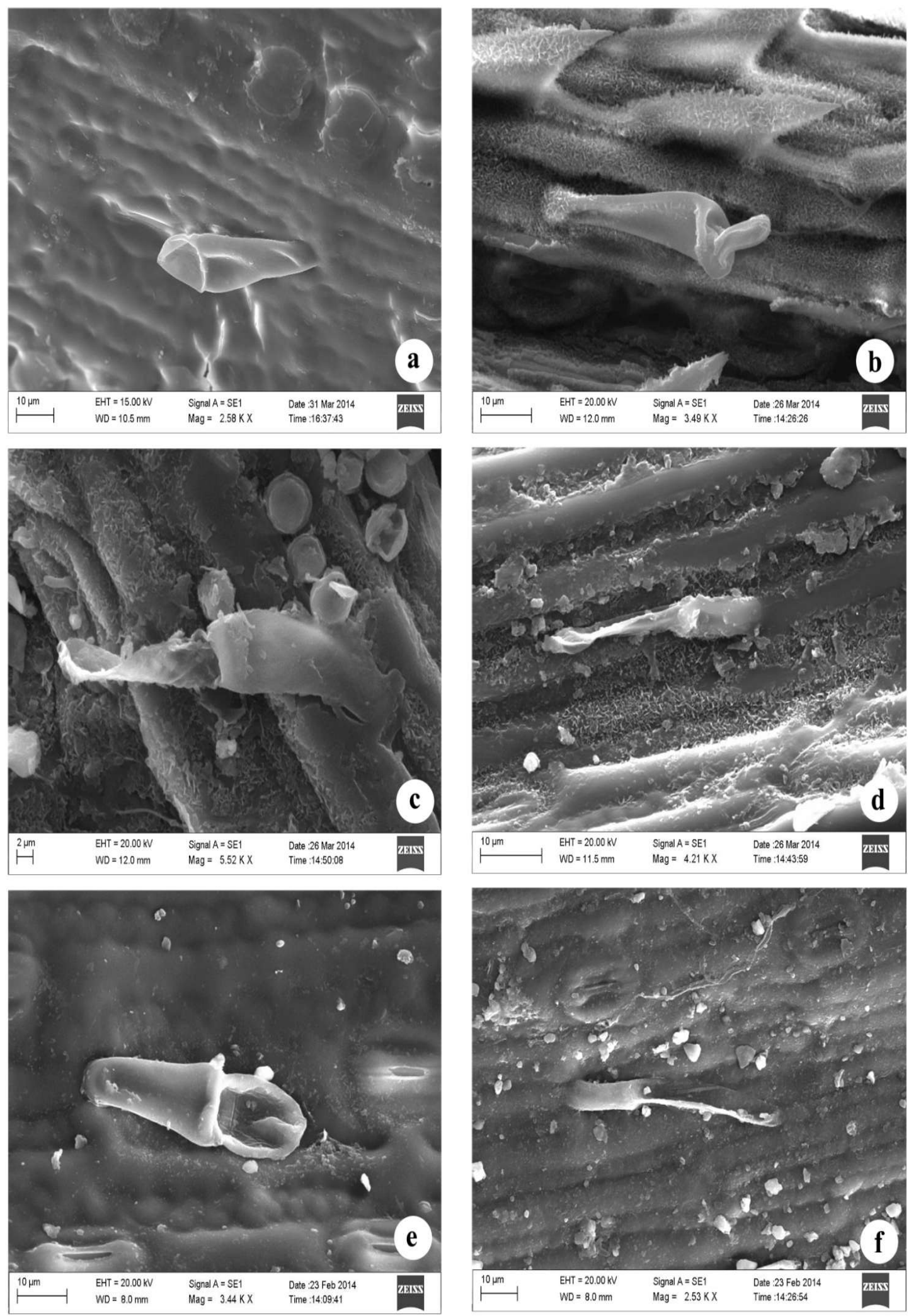

Plate 5. SEM feature of microhairs of: a. E. tef (Zucc.) Trotter; b. E. tenuifolia (A. Rich.) Hochst. ex Steud.; c. E. tremula Hochst. ex Steud.; d. E. unioloides (Retz.) Nees ex Steud.; e. E. viscosa (Retz.) Trin.; f. E. zeylanica Nees \& Mey. 


\section{CONCLUSION}

Though few species of Eragrostis were characterized by panicoid and ennaepogon (intermediate) types of microhairs, rest of them were found to have chloridoid type of microhairs. This character could be used successfully for the delimitation of species of Eragrostis in India when used along with other morphological characters.

\section{ACKNOWLEDGEMENT}

The authors are grateful to the Ministry of Environment, Forests and Climate Change for providing the financial assistance under All India co-ordinated Project in Taxonomy (AICOPTAX). They are also thankful to the Director, Botanical Survey of India, Kolkata, for providing facilities and encouragement.

\section{REFERENCES}

AMARASINGHE, V. AND L. WATSON. 1990. Taxonomic significance of microhair morphology in the Genus Eragrostis Beauv. (Poaceae). Taxon. 39 (1): 59-65.

BOR, N. L. 1960. The Grasses of Burma, Ceylon, India and Pakistan (excluding Bambuseae). Pergamon Press, London.

CLAYTON, W. D. AND S. A. RENVOIZE. 1986. Genera Graminum, grasses of the world. Kew Bulletin Additional Series. 13: 1-389.

GIRALDO-CANAS, D., P. M. PETERSON AND I. SANCHEZ VEGA. 2012. The genus Eragrostis (Poaceae: Chloridoideae) in northwestern South America (Colombia, Ecuador, and Peru): Morphological and taxonomic studies. Bibl. Jose Jeron.Trian. 23: 1-180.

INGRAM, A. L. 2010. Evolution of leaf blade anatomy in Eragrostis (Poaceae). Syst. Bot. 35(4): 755-765.

JOHNSTON, C. R. AND L. WATSON. 1976. Microhairs: A universal characteristic of non-festucoid grass genera? Phytomorphology. 26: 297-301.

KARTHIKEYAN, S., S. K. JAIN, M. P. NAYAR AND M. SANJAPPA. 1989. Poaceae. In: Florae Indicae Enumeratio Monocotyledonae. Botanical Survey of India, Calcutta.

TATEOKA, T., S. INOWE AND K. KAWANO. 1959. Notes on some grasses IX: Systematic significance of bicellular microhairs of leaf epidermis. Bot. Gazz. 121(2): 80-91.

METCALFE, C. R. 1960. Anatomy of the Monocotyledons I. Gramineae. Oxford University, London.

VELDKAMP, J. F. 2002. Revision of Eragrostis (Gramineae, Chloridoideae) in Malesia. Blumea 47: 157-204.

VIVEK, C. P., G.V.S. MURTHY AND V. J. NAIR. 2012. A new species Eragrostis henryi (Poaceae: Eragrostideae) from Tamil Nadu, India. Nelumbo 54: 9- 12.

VIVEK, C. P., G.V.S. MURTHY AND V. J. NAIR. 2013a. Eragrostis cilianensis (Poaceae: Eragrostideae): A new species from the hills of Kerala and Tamil Nadu, India. Indian J. Forestry 36 (3): 401-404.

VIVEK, C. P., G. V. S. MURTHY AND V. J. NAIR. 2013b. Eragrostis jainii (Poaceae: Eragrostideae) A new species from Kerala, India. Nelumbo 55:1- 5.

VIVEK, C. P., G. V. S. MURTHY AND V. J. NAIR. 2013c. Eragrostis nilgiriensis sp. nov. (Poaceae) from Nilgiri district, Tamil Nadu, India. Nord. J. Bot. 31: 700-703.

WATSON, L., H. T. CLIFFORD AND M. J. DALLWITZ. 1985. The classification of Poaceae: subfamilies and subtribes. Australian J. Bot. 33: 433-484. 